\title{
Comparação de técnicas no diagnóstico enteroparasitário em pacientes HIV positivo
}

\section{no alto pantanal}

\author{
Comparison of techniques in enteroparasitary diagnosis in HIV positive patients at the high
} pantanal

Comparación de técnicas de diagnóstico enteroparasitario en pacientes VIH positivos del pantanal superior

Recebido: 16/06/2021 | Revisado: 22/06/2021 | Aceito: 29/06/2021 | Publicado: 13/07/2021

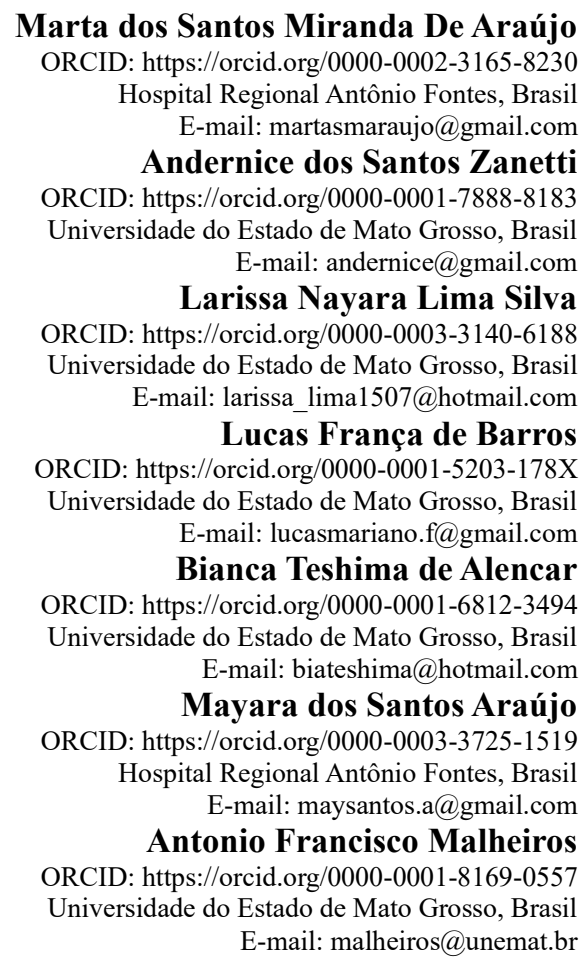

\begin{abstract}
Resumo
O objetivo desse estudo foi analisar frequencia de amostras positivas entre técnicas coprológicas no diagnóstico de enteroparasitas em pacientes portadores do vírus HIV no Alto Pantanal. Foram coletadas e analisadas amostras fecais de 28 pacientes portadores do vírus HIV, em que as técnicas utilizadas foi a de sedimentação espontânea, método de centrífugo-flutuação em solução de sacarose e a técnica com coloração de Safranina. Na técnica de Hoffman a maior positividade de enteroparasitas foi a observada para o protozoário Blastocystis spp. comum a frequência de 52,38\%, seguido por Giardia lamblia com 21,47\%. Em Sheather a maior positividade também foi Blastocystis spp. 63,8\%, seguido por Giardia lamblia 33,33\%. A técnica utilizando o corante de Safranina possível identificação de quatro protozoários que não foram visualizados nas técnicas de sedimentação e flutuação. O princípio que diferem as técnicas de flutuação e sedimentação no diagnóstico não condiz com os resultados observados nesse estudo, tendo em vista que ambas as técnicas apresentaram eficiência na detecção de protozoários, como o Blastocystis spp. e Giardia lamblia, independentemente de estarem ou não em soluções saturadas. Sendo assim, para garantir melhor resultado, a associação de técnicas resulta sempre em maior confiabilidade, principalmente quando se utilizam técnicas de fundamentos diferentes.
\end{abstract}

Palavras-chave: Hoffman; Sheather; Safranina; Exame coproparasitológico; Pacientes imunodeficiêntes.

\section{Abstract}

The objective of this study was to analyze the efficiency and agreement between coprological techniques in the diagnosis of enteroparasites in patients with HIV in the Upper Pantanal. Fecal samples were collected and analyzed from 28 patients with the HIV virus, in which the techniques used were spontaneous sedimentation, the centrifugal-fluctuation 
method in sucrose solution and the technique with Safranin staining. In Hoffman's technique, the greatest positivity of enteroparasites was that observed for the protozoan Blastocystis spp. the frequency of $52.38 \%$ is common, followed by Giardia lamblia with $21.47 \%$. In Sheather the greatest positivity was also Blastocystis spp. $63.8 \%$, followed by Giardia lamblia $33.33 \%$. The technique using the Safranina dye possible identification of four protozoa that were not visualized in the sedimentation and flotation techniques. The principle that the flotation and sedimentation techniques differ in the diagnosis is not consistent with the results observed in this study, given that both techniques were efficient in detecting protozoa, such as Blastocystis spp. and Giardia lamblia, regardless of whether or not they are in saturated solutions. Therefore, to ensure better results, the association of techniques always results in greater reliability, especially when using different fundamental techniques.

Keywords: Hoffman; Sheather; Safranina; Coproparasitological examination; Immunodeficient patients.

\section{Resumen}

El objetivo de este estudio fue analizar la frecuencia de muestras positivas entre técnicas coprológicas en el diagnóstico de parásitos intestinales en pacientes con el virus del VIH en el Alto Pantanal. Se recolectaron y analizaron muestras de heces de 28 pacientes con el virus del VIH, en las cuales las técnicas empleadas fueron la sedimentación espontánea, el método de centrifugación-flotación en solución de sacarosa y la técnica con tinción con safranina. En la técnica de Hoffman, la mayor positividad de enteroparásitos se observó para el protozoo Blastocystis spp. común la frecuencia de $52,38 \%$, seguida de Giardia lamblia con 21,47\%. En Sheather, la positividad más alta también fue Blastocystis spp. $63,8 \%$, seguida de Giardia lamblia 33,33\%. La técnica que utiliza el colorante Safranina permite la identificación de cuatro protozoos que no fueron visualizados en las técnicas de sedimentación y flotación. El principio de que las técnicas de fluctuación y sedimentación difieren en el diagnóstico no concuerda con los resultados observados en este estudio, considerando que ambas técnicas fueron eficientes en la detección de protozoos, como Blastocystis spp. y Giardia lamblia, independientemente de si se encuentran en soluciones saturadas o no. Por tanto, para asegurar un mejor resultado, la asociación de técnicas siempre da como resultado una mayor fiabilidad, especialmente cuando se utilizan técnicas con diferentes fundamentos.

Palabras clave: Hoffman; Sheather; Safranina; Examen de heces; Pacientes inmunodeficientes.

\section{Introdução}

A relação entre parasito e hospedeiro se estabelece por meio de absorção da base nutricional e/ou energéticas podendo ocasionar lesões (Wilson, 1980; Mascarini, 2003), sendo mais frequente em populações carentes e mais agressivas em pacientes imunodeficientes (Leite \& Waissmann, 2012).

A recuperação e a identificação nos seus diferentes estágios de desenvolvimento na análise coproparasitológica, podem ser por meio da utilização de alguns métodos de concentração ou coloração (Sant'anna; Oliveira \& Melo, 2013), como a sedimentação espontânea (como o Hoffman), técnica de Sheather e o método de coloração com Safranina.

O uso de diferentes métodos laboratoriais para diagnosticar doenças ocasionadas por parasitas são importantes não só pela frequência de infecções enteroparasitárias que comprometem a saúde humana (Mariano et al., 2005), mas também para se obter maior eficiência e consequentemente, melhor diagnóstico para o tratamento nos grupos de maior risco de contágio e mais suscetíveis às agressões ocasionadas pela relação entre o parasita e hospedeiro.

A busca por laboratórios de diagnóstico clinico por métodos que combine a especificidade, sensibilidade, velocidade e baixo custo operacional tem sido frequente. Ao combinar esses requisitos com o exame coproparasitológico de forma correta, se torna possível a identificação do agente etiológico podendo ser usado em estudos epidemiológicos como orientação para o desenvolvimento de medidas profiláticas, assim como um possível indicador da qualidade da saúde da população (Carli, 2001; Mendes et al., 2005; Machado et al., 2008; Rezende et al., 2015).

Acredita-se que o uso diversificado de exames coproparasitológicos em laboratórios aumentam o espectro de diagnóstico possibilitando um melhor tratamento dos pacientes sendo necessário testes de eficiência e análises comparativas entre os diferentes métodos utilizados. Sendo assim, o objetivo desse estudo foi analisar a eficiência e a concordância entre técnicas coprológicas no diagnóstico de enteroparasitas em pacientes portadores do vírus HIV no Alto Pantanal. 


\section{Metodologia}

\subsection{Tipos de estudo}

A pesquisa caracteriza-se como transversal com base em fonte primária e amostra não probabilística.

Trata-se de um estudo transversal por ser um estudo epidemiológico no qual fator e efeito é observada num mesmo momento histórico (Rouquayrol, 1999).

\subsection{Descrição do local de estudo}

O município de Cáceres Mato Grosso, localiza -se na microrregião do Alto Paraguai e distante aproximadamente 215 km da capital Cuiabá. Possui uma população de aproximadamente 87.942 habitantes e está distante cerca de 100 quilômetros da fronteira do Brasil com a Bolívia, possui uma área territorial de 24.965,94 Km². (IBGE, 2010).

A região de Cáceres apresenta um clima tropical com estações bem definidas: seca no inverno e úmida no verão. O período de janeiro a março é o mais úmido, e o período entre setembro e outubro é o de maior índice de radiação solar e de mais baixa precipitação. (Aguinaldo et al., 2008).

\subsection{População/amostra do Estudo}

A população de estudo da presente pesquisa foi composta por 28 pacientes portadores do vírus HIV e acompanhados no SAE/CTA, que aceitaram participar da pesquisa assinando o Termo de Consentimento Livre e Esclarecido - TCLE, que residem no município de Cáceres- MT e atenderam aos critérios de inclusão, que foram:

- $\quad$ Ser portador do vírus HIV;

- Residir no município Cáceres;

- Ter idade acima de 18 anos.

\subsection{Técnicas de análises parasitológicas e coloração com Safranina}

A análise parasitológica das amostras foi realizada por meio da coleta de material fecal em potes coletores universais, rotulados adequadamente e transportadas para o laboratório de análises parasitológicas da Universidade do Estado de Mato Grosso (UNEMAT), onde foram armazenados a $4^{\circ} \mathrm{C}$.

Foram realizadas duas técnicas convencionais de análises coprológicas em todas amostras coletadas, o método de sedimentação espontânea (Hoffman et al., 1923) e método de centrífugo-flutuação em solução de sacarose (Sheather, 1923); e a técnica com coloração de Safranina.

\subsection{Considerações éticas}

O projeto foi submetido ao comitê de ética e pesquisa da Universidade do Estado de Mato Grosso (UNEMAT), para atender à resolução do Conselho Nacional de Saúde no 466, de 12 de dezembro de 2012, que regulariza os estudos envolvendo seres humanos e foi aprovado sob o Parecer $n^{\circ} 3.323 .211$.

\section{Resultados}

\subsection{Prevalência de infecções enteroparasitárias na população estudada}

Referente a prevalência de enteroparasitas segundo técnicas de analises coprológicas dos pacientes HIV (Tabela 1), observou-se que em cada uma das técnicas utilizadas, apresentou uma prevalência diferente entre os achados parasitológicos, destacando que Cryptosporidium spp. foi identificado somente em Sheather $(42,86 \%)$ e Safranina $(64,29 \%)$. Giardia lamblia 
apareceu somente em Hoffman (32,14\%) e Sheather (3,57\%). Alguns enteroparasitas foram identificados somente na técnica de Safranina como Entamoeba histolytica (3,57\%), Cyclospora spp. (75\%), Microsporidium spp. (3,57\%) e Isospora belli (14,29\%). Outros enteroparasitas como Endolimax nana (21, 43\%), Chilomastix mesnili (14,29\%) e Strongyloids stercoralis (3,57\%) somente em Hoffman.

Tabela 1. Prevalência de enteroparasitas segundo técnicas de analises coprológicas dos pacientes HIV positivos residentes no município de Cáceres-MT, no ano de 2019.

\section{Prevalência HIV (\%)}

\begin{tabular}{lccc}
\hline & Hoffman & Sheather & Safranina \\
Protozoário & & 82,14 & 3,57 \\
Blastocystis spp. & 78,57 & 42,86 & 64,29 \\
Cryptosporidium spp. & $\mathrm{n}^{*}$ & 3,57 & $\mathrm{n}^{*}$ \\
Giardia lamblia & 32,14 & $\mathrm{n}^{*}$ & 3,57 \\
Entamoeba histolytica & $\mathrm{n}^{*}$ & $\mathrm{n}^{*}$ & $\mathrm{n}^{*}$ \\
Endolimax nana & 21,43 & $\mathrm{n}^{*}$ & 75,00 \\
Cyclospora spp. & $\mathrm{n}^{*}$ & $\mathrm{n}^{*}$ & 3,57 \\
Microsporidium spp. & $\mathrm{n}^{*}$ & $\mathrm{n}^{*}$ & 14,29 \\
Isospora belli & $\mathrm{n}^{*}$ & $\mathrm{n}^{*}$ & $\mathrm{n}^{*}$ \\
Chilomastix mesnili & 14,29 & & $\mathrm{n}^{*}$ \\
Helmintos & & & $\mathrm{n}^{*}$ \\
Strongyloides stercoralis & 3,57 & & \\
\hline
\end{tabular}

Legenda: $\mathrm{n}^{*}=$ não observado.

Fonte: Autores (2021).

\subsection{Comparação de desempenho dos diferentes métodos de diagnóstico de parasitas intestinais}

Quanto à frequência de enteroparasitas por técnicas de análises utilizadas, observou que para Blastocystis spp. a melhor técnica de análise foi Sheather, com uma frequência de positividade de 63,89\% (N=23) seguido de Hoffman com 52,38\% (N=22); para Cryptosporidium spp. foi a técnica de Safranina com 39,13\% (N=18) seguido Sheather 33,33\% (N=12); para Giardia lamblia foi a técnica de Hoffman com 21,43\% ( $=9$ ) seguido de Sheather com 2,78\% (N=1) (Tabela 2).

Alguns enteroparasitas foram identificados somente pela técnica de Safranina como Entamoeba histolytica e Microsporidium spp. com 2,17\% (N=1); Cyclospora spp. com 45,65\% ( $\mathrm{N}=21)$ e Isospora belli com 8,70\% (N=4). E outros enteroparasitas apresentaram positividade somente pela técnica de Hoffman sendo estes Endolimax nana com 14,29\% (N=6); Chilomastix mesnili com 9,52\% (N=4) e Strongyloides stercoralis da classe dos helmintos com 2,38\% (N=1). 
Tabela 2. Comparação dos métodos parasitológicos no diagnóstico de helmintos e protozoários intestinais nos 28 pacientes HIV residentes no município de Cáceres-MT, no ano de 2019.

\begin{tabular}{|c|c|c|c|c|c|c|}
\hline \multirow{2}{*}{ Parasita } & \multicolumn{2}{|c|}{ Hoffman } & \multicolumn{2}{|c|}{ Sheather } & \multicolumn{2}{|c|}{ Coloração } \\
\hline & $\mathbf{N}$ & $\%$ & $\mathbf{N}$ & $\%$ & $\mathbf{N}$ & $\%$ \\
\hline \multicolumn{7}{|l|}{ Protozoários } \\
\hline Blastocystis spp. & 22 & 52,38 & 23 & 63,89 & 1 & 2,17 \\
\hline Cryptosporidium spp. & 0 & 0,00 & 12 & 33,33 & 18 & 39,13 \\
\hline Giardia lamblia & 9 & 21,43 & 1 & 2,78 & 0 & 0,00 \\
\hline Entamoeba histolytica & 0 & 0,00 & 0 & 0,00 & 1 & 2,17 \\
\hline Endolimax nana & 6 & 14,29 & 0 & 0,00 & 0 & 0,00 \\
\hline Cyclospora spp. & 0 & 0,00 & & 0,00 & 21 & 45,65 \\
\hline Microsporidium spp. & 0 & 0,00 & 0 & 0,00 & 1 & 2,17 \\
\hline Isospora belli & 0 & 0,00 & 0 & 0,00 & 4 & 8,70 \\
\hline Chilomastix mesnili & 4 & 9,52 & 0 & 0,00 & 0 & 0,00 \\
\hline \multicolumn{7}{|l|}{ Helmintos } \\
\hline Strongyloides stercoralis & 1 & 2,38 & 0 & 0,00 & 0 & 0,00 \\
\hline Total & 42 & 100 & 36 & 100 & 46 & 100 \\
\hline
\end{tabular}

Fonte: Autores (2021).

A Tabela 3 reforça e detalha os achados referente à positividade enteroparasitária que cada técnica parasitológica identificou nos pacientes HIV. 
Tabela 3. Resultados dos métodos parasitológicos no diagnóstico de helmintos e protozoários intestinais nos pacientes HIV residentes no município de Cáceres-MT, no ano de 2019.

\begin{tabular}{|c|c|c|c|}
\hline RESUTADO & HOFFMAM & SAFRANINA & SHEATHER \\
\hline POSITIVO & Blastocystis spp. & Blastocystis spp, Cryptosporidium spp, Isospora belli & Blastocystis spp, Cryptosporidium spp \\
\hline POSITIVO & S. stercoralis, Blastocystis spp., C. mesnili, E.nana & Cryptosporidium spp, Isospora belli, Microsporidium spp & Blastocystis spp, Cryptosporidium spp \\
\hline POSITIVO & Blastocystis spp. & Cryptosporidium spp, Isospora belii, Cyclospora spp & Blastocystis spp, Cryptosporidium spp \\
\hline POSITIVO & Blastocystis spp. & Cyclospora spp & Blastocystis spp \\
\hline POSITIVO & Blastocystis spp., E.nana & Cyclospora spp,Isospora belli, & Blastocystis spp, Cryptosporidium spp \\
\hline POSITIVO & Giardia lamblia & Cryptosporidium spp, Cyclospora spp & Blastocystis spp, Cryptosporidium spp \\
\hline POSITIVO & Blastocystis spp. & Cryptosporidium spp, Cyclospora spp & Blastocystis spp, Cryptosporidium spp \\
\hline POSITIVO & Giardia lamblia, Blastocystis spp. & Cryptosporidium spp, Cyclospora spp & Blastocystis spp, Cryptosporidium spp \\
\hline POSITIVO & Giardia lamblia, Blastocystis spp. & Cyclospora spp & Giardia spp \\
\hline POSITIVO & Giardia lamblia, Blastocystis spp. & Cryptosporidium spp, Cyclospora spp & Blastocystis spp \\
\hline POSITIVO & Blastocystis spp. & Cyclospora spp & Cryptosporidium spp \\
\hline POSITIVO & Giardia lamblia, Blastocystis spp. & Negativa & Negativa \\
\hline POSITIVO & Giardia lamblia, Blastocystis spp. & Cyclospora spp & Blastocystis spp \\
\hline POSITIVO & Giardia lamblia, Blastocystis spp., E.nana, C.mesnili & Cryptosporidium spp & Cryptosporidium spp \\
\hline POSITIVO & Giardia lamblia, Blastocystis spp. & Negativa & Blastocystis spp \\
\hline POSITIVO & Giardia lamblia, Blastocystis spp. & Cryptosporidium spp, Cyclospora spp & Blastocystis spp, Cryptosporidium spp \\
\hline POSITIVO & Negativa & Cryptosporidium spp, Cyclospora spp & Negativa \\
\hline POSITIVO & Blastocystis spp, E. nana, C. mesnili & Cryptosporidium spp, Cyclospora spp, E. histolytica & Blastocystis spp \\
\hline POSITIVO & C. mesnili & Cryptosporidium spp, Cyclospora spp & Blastocystis spp, Cryptosporidium spp \\
\hline POSITIVO & E. nana & Cryptosporidium spp, Cyclospora spp & Blastocystis spp \\
\hline
\end{tabular}


Research, Society and Development, v. 10, n. 8, e30510817262, 2021

(CC BY 4.0) | ISSN 2525-3409 | DOI: http://dx.doi.org/10.33448/rsd-v10i8.17262

\begin{tabular}{|c|c|c|c|}
\hline POSITIVO & Blastocystis spp & Cryptosporidium spp, Cyclospora spp & Blastocystis spp \\
\hline POSITIVO & Blastocystis spp & Cryptosporidium spp, Cyclospora spp & Blastocystis spp \\
\hline POSITIVO & Negativa & Cyclospora spp & Blastocystis spp \\
\hline POSITIVO & Blastocystis spp & Cryptosporidium spp & Blastocystis spp, Cryptosporidium spp \\
\hline POSITIVO & Blastocystis spp & Cryptosporidium spp, Cyclospora spp & Blastocystis spp \\
\hline POSITIVO & Negativa & Negativa & Blastocystis spp \\
\hline POSITIVO & Blastocystis spp & Cryptosporidium spp, Cyclospora spp & Blastocystis spp \\
\hline POSITIVO & Blastocystis spp, E. nana & Cryptosporidium spp, Cyclospora spp & Blastocystis spp \\
\hline
\end{tabular}

Fonte: Autores (2021). 
De acordo com a Figura 2, é possível observar a efetividade de cada técnica testada para leitura de positividade enteroparasitária em que o Hoffman foi possível identificar Blastocystis spp. G. lambria e E. nana; a técnica de Sheather Blastocystis spp. e Cryptosporidium spp.; e por último a coloração de Safranina para Cryptosporidium spp. e Cyclospora spp.

Figura 1. Comparação entre as técnicas utilizadas para análises das amostras parasitológicas de helmintos e protozoários intestinais nos pacientes HIV.

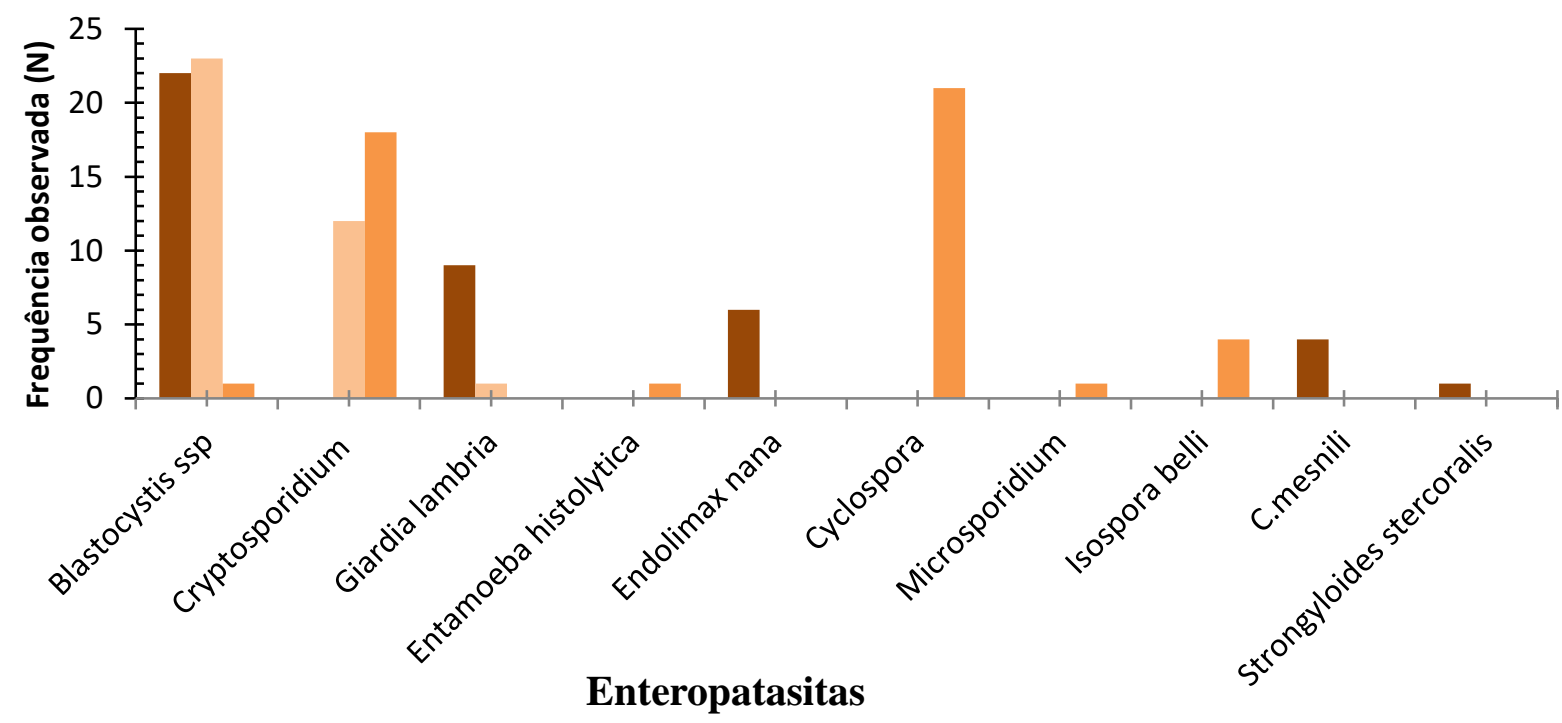

- Hoffman $\quad$ Sheather $\quad$ Coloração

Giardia

lamblia foram a de Hoffman e Sheather, entretanto, a técnica de Hoffman mostrou-se mais sensível para o diagnóstico desses protozoários apresentando maior positividade no diagnóstico. Essa falta de concordância nos métodos também foi observada em um estudo de Eymael et al., (2010), que em 20 amostras observou diferença estatística significativa entre as duas técnicas de sedimentação (Eymael; Schuh \& Tavares, 2010).

Ao comparar técnicas de flutuação (como a de Sheather) com técnicas de sedimentação (Hoffman), elas possuem princípios de detecção parasitária distintas em que o método de flutuação facilita a identificação dos ovos de nematóides e oocistos de protozoários em soluções saturadas. Por outro lado, o método de sedimentação auxilia na observação de ovos pesados que não flutuam em soluções saturadas, como é o caso de ovos de trematódeos e de alguns cestóides (Sloss et al., 1999; Táparo et al., 2006).

Esse princípio que diferem as técnicas de flutuação e sedimentação no diagnóstico não condiz com os resultados observados nesse estudo, tendo em vista que ambas as técnicas apresentaram eficiência na detecção de protozoários, como o Blastocystis spp. e Giardia lamblia, independentemente de estarem ou não em soluções saturadas.

A técnica de Sheather apresentou um índice de prevalência melhor para os protozoários, conforme estudos de Malheiros (2011), sua pesquisa evidenciou uma boa sensibilidade desta técnica para a detecção de cistos de Giardia lamblia e outros protozoários, mostrando que é uma técnica viável a ser utilizada.

Na técnica de Safranina (coloração) houve uma prevalência significativa para enteroparasitas como Cryptosporidium spp., Cyclospora spp. e Isospora belli. A técnica de coloração mostrou ser eficiente para o diagnóstico desses enteroparasitas, visto que Cryptosporidium spp. apresentou concordância com a técnica de Sheather. Valentim (2011) também avaliou a técnica de coloração como sendo uma boa opção para o diagnóstico de protozoários em seus estudos realizados no Instituto Fernandes 
Figueira/ FIOCRUZ, que obteve uma porcentagem de positividade de $57 \%$ sobre o total de 50 amostras para oocistos de Cryptosporidium spp. através da técnica utilizando safranina azul de metileno, comprovando assim a eficácia da técnica para o diagnóstico.

Técnicas de coloração não são ainda rotina para a maioria dos laboratórios clínicos, porém a demonstração da esporulação fornece definitiva evidência do diagnóstico (Mezzari; Antunes \& Wiebbelling, 1999). Ghoshal et al. (2018) em sua pesquisa na Índia, diz que os métodos de coloração continuam sendo o esteio de diagnóstico na maioria laboratórios no mundo todo, tendo um custo baixo em seus reagentes

Bogaerts et al. (1984) em um estudo na África central, comparou duas técnicas de coloração, e afirma que as técnicas simples de coloração é possível, sugerindo que a rotina para o exame de Cryptosporidium spp. deve fazer parte da rotina parasitológica e que o método de Safranina tem uma boa especificidade.

Algumas espécies como o Cryptosporidium parvum e Cystoisopora belli atuam claramente como oportunistas provocando quadros de enterite em 15 a 50\% dos pacientes com AIDS (Soave \& Jonhson, 1988; Siddiqui et al., 2007; Rossit et al., 2007).

A escolha de técnicas para serem implementadas na rotina laboratorial no setor de Parasitologia envolve vários fatores. Cabe lembrar que, para garantir melhor resultado, a associação de técnicas resulta sempre em maior confiabilidade, principalmente quando se utilizam técnicas de fundamentos diferentes (Alencar et al., 2020).

São necessárias técnicas laboratoriais adequadas que apresentem boa sensibilidade, especificidade e reprodutibilidade. A avaliação da precisão de diferentes técnicas parasitológicas permite determinar a concordância e a reprodutibilidade para aplicação em laboratórios (Lima et al., 2018).

\section{Conclusão}

As três técnicas utilizadas são de baixo custo e evidenciou uma boa eficácia para o diagnóstico das enteroparasitoses. É preciso ter no mínimo três técnicas para que possa comprovar diagnósticos parasitológicos de fezes nesta população, garantindo assim um diagnóstico ideal.

\section{Referências}

Alencar, B. T., dos Santos Zanetti, A., Vilella, S. H., de Araújo, M. D. S. M., Silva, L. N. L., de Alencar, R. T., \& Malheiros, A. F. (2020). Fatores socioambientais e prevalência de enteroparasitas em pacientes em hemodiálise no pantanal mato-grossense, Brasil. Research, Society and Development, 9(10), e5109108738e5109108738.

Bogaerts, J., Lepage, P., Rouvroy, D., \& Vandepitte, J. (1984). Cryptosporidium spp., a frequent cause of diarrhea in Central Africa. Journal of Clinical Microbiology, 20(5), 874-876.

Eymael, D., Schuh, G. M., \& Tavares, R. G. (2010). Padronização do diagnóstico de Blastocystis hominis por diferentes técnicas de coloração. Revista da Sociedade Brasileira de Medicina Tropical, 43(3), 309-312.

Ghoshal, U., Jain, V., Dey, A., \& Ranjan, P. (2018). Evaluation of enzyme linked immunosorbent assay for stool antigen detection for the diagnosis of cryptosporidiosis among HIV negative immunocompromised patients in a tertiary care hospital of northern India. Journal of infection and public health, 11(1), 115-119.

Hoffman, W. A., Pons, J. A., \& Janer, J. L. (1934). The sedimentation-concentration method in schistosomiasis mansoni. J Public Health Trop Med, 9, 283-91, 1934.

IBGE. Instituto Brasileiro de Geografia e Estatística. (2010) Censo demográfico 2010 http://www.censo2010.ibge.gov.br

Leite, L. H. M., \& Waissmann, W. (2012). Enteroparasitoses em pacientes ambulatoriais portadores de HIV/AIDS e abastecimento domiciliar de água. Revista de Ciências Médicas, 13(4).

Lima, J. A. S., Rezende, H. H. A., Rocha, T. M. D. D., \& Castro, A. M. D. (2018). Analysis of the accuracy of different laboratory methods for the diagnosis of intestinal parasites from stray and domiciled cats (Felis catus domesticus) in Goiânia, Goiás, Brazil. Revista Brasileira de Parasitologia Veterinária, 27(1), 9497. 
Machado, E. R., Santos, D. S., \& Costa-Cruz, J. M. (2008). Enteroparasites and commensals among children in four peripheral districts of Uberlândia, State of Minas Gerais. Revista da Sociedade Brasileira de Medicina Tropical, 41(6), 581-585.

Malheiros, A. F. (2011). Ocorrência de patógenos intestinais e fatores de risco associados à infecção entre os índios tapirapé habitantes da Amazônia MatoGrossense, Brasil (Doctoral dissertation, Universidade de São Paulo).

Mascarini, L. M. (2003). Uma abordagem histórica da trajetória da parasitologia. Ciência \& Saúde Coletiva, 8, 809-814.

Trajano, F. D. S., Pinto, A. D. S., Ferreira, A. C., Kato, C. M. D. B., Cunha, R. B., \& Viana, F. M. (2000). Estudo comparativo entre os métodos de análise cefalométrica manual e computadorizada. Rev. dent. press ortodon. ortop. maxilar, 57-62.

Mezzari, A., Antunes, H. B. B., \& Wiebbelling, A. M. P. (1999). Cyclospora cayetanensis, um novo protozoário a ser pesquisado. Revista da Associação Médica Brasileira, 45(4), 347-348.

Neves, L. A. D. S., Canini, S. R. M., Reis, R. K., Santos, C. B. D., \& Gir, E. (2012). Sida y tuberculosis: la coinfección vista según la perspectiva de la calidad de vida de los individuos. Revista da Escola de Enfermagem da USP, 46(3), 704-710.

Rezende, H. H. A., Avelar, J. B., Storchilo, H. R., Vinaud, M. C., \& Castro, A. M. D. (2015). Evaluation of the accuracy of parasitological techniques for the diagnosis of intestinal parasites in cats. Revista Brasileira de Parasitologia Veterinária, 24(4), 471-474.

Rossit, A. R. B., de Almeida, M. T. G., Nogueira, C. A. M., da Costa Oliveira, J. G., Barbosa, D. M. U., Moscardini, A. C., \& Machado, R. L. D. (2007). Bacterial, yeast, parasitic, and viral enteropathogens in HIV-infected children from Sao Paulo State, Southeastern Brazil. Diagnostic microbiology and infectious disease, 57(1), 59-66.

Rouquayrol, M. Z., \& Gurgel, M. (2021). Rouquayrol: epidemiologia e saúde. Medbook.

Sant'Anna, L. M. L., de Jesus Oliveira, F., \& de Melo, C. M. (2013). Estudo comparativo de técnicas parasitológicas baseada no princípio de sedimentação espontânea (Hoffman) e Parasitokitß. Scire Salutis, 3(1), 6-15.

Sheather, A. L. (1923). The detection of intestinal protozoa and mange parasites by a floatation technique. Journal of Pathology and Therapy, 36(pt. 4).

Siddiqui, U., Bini, E. J., Chandarana, K., Leong, J., Ramsetty, S., Schiliro, D., \& Poles, M. (2007). Prevalence and impact of diarrhea on health-related quality of life in HIV-infected patients in the era of highly active antiretroviral therapy. Journal of clinical gastroenterology, 41(5), 484-490.

Silva, A., Souza Filho, E. D., \& Cunha, S. D. (2008). Padrões de canal do rio Paraguai na região de Cáceres (MT). Revista Brasileira de Geociências, 38(1), 167-177.

Sloss, W. M.; Zajac, M. A. \& Kemp, R. L.(1999) Parasitologia clínica veterinária. Manole.

Soave, R., \& Johnson Jr, W. D. (1988). Cryptosporidium and Isospora belli infections. Journal of Infectious Diseases, 157(2), 225-229.

Táparo, C. V., Perri, S. H., Serrano, A. C. M., Ishizaki, M. N., Costa, T. P., do Amarante, A. F., \& Bresciani, K. D. (2006). Comparação entre técnicas coproparasitológicas no diagnóstico de ovos de helmintos e oocistos de protozoários em cães. Revista Brasileira de Parasitologia Veterinária, 15(1), 1-5.

Nascimento Valentim, T. S., \& Cardozo, S. V. (2011). Avaliação qualitativa de oocistos de Cryptosporidium sp. em amostras fecais de pacientes atendidos no Instituto Fernandes Figueira/Fiocruz. Saúde \& Ambiente em Revista, 6(1), 11-16.

Carli, G. A. (2001). Parasitologia clínica: seleção de métodos e técnicas de laboratório para diagnóstico das parasitoses humanas. In Parasitologia clínica: seleção de métodos e técnicas de laboratório para diagnóstico das parasitoses humanas (pp. 810-810).

Wilson, R. A. (1980). Introdução à parasitologia. EPU. 